\title{
Indexing PXRD Structural Parameters of Graphene Oxide-Doped Metal-Organic Frameworks
}

\author{
Khaliesah Kamal, Mohamad Azmi Bustam, Azmi Mohd Shariff, Pascaline Pré, Lomig Hamon
}

\begin{abstract}
A family of metal-organic framework, $\left[\mathrm{Mg}_{3} \mathrm{O}_{3}\left(\mathrm{CO}_{2}\right)_{3}\right]_{\infty}$ nanocrystallites which are also known as Mg-MOF-74, were synthesized by solvothermal method at high temperature under autogenous pressure. The graphene oxide was embedded into the framework via in-situ synthesis method to form a composite structure of Mg-MOF-74@nGO. $n$ was varied from 0.1 to 0.6 wt\% of the weight of pristine material. The synthesized samples were examined under Powder X-Ray Diffraction analysis to affirm their crystalline structure. Further studies were conducted by indexing the lattice parameters of the hexagonal crystals. The volume of unit cell and Scherrer's crystallite size were also determined to study the effect of GO loading amount on their grown dimensions. The results showed that the presence of $G O$ in the range of 0.1-0.6 wt\% did not influence the dimension of the crystal except a slight expansion in vertical direction with the increment of GO loading.
\end{abstract}

Index Terms: Indexing, PXRD, Metal-Organic Framework, Graphene Oxide, Composites, Crystals.

\section{INTRODUCTION}

Metal-organic framework (MOF) is the assembly of strong-coordinated molecular building blocks which are formed from the bonding between central metal ions or clusters and bridging organic linkers. The repeating crystalline structures make them easier to be characterized when compared to amorphous solids. This nanostructured material offers the liberty to adjust its porosity and functionality by changing metal ions or linkers. The structure can be modified to suit the specific application including gas capture and storage, gas sensor, luminescence, magnetic material and drug delivery.

Different metal nodes can be used to produce MOF including transition metals, alkaline earth elements, p-block elements, actinides and even mix metals [1]. Meanwhile, possible linkers that can be used to bind the metal ions are polycarboxylates, phosphonates, sulfonates, imidazolates, amines, pyridyl and phenolates [2]. The most commonly used is carboxylate-based linkers of which the chemical

Revised Manuscript Received on September 22, 2019.

Khaliesah Kamal, Department of Chemical Engineering, Universiti Teknologi PETRONAS, 31750 Seri Iskandar, Malaysia. and Department of Energy Systems and Environment, IMT Atlantique, GEPEA CNRS, 44300 Nantes, France

Mohamad Azmi Bustam, Department of Chemical Engineering, Universiti Teknologi PETRONAS, 31750 Seri Iskandar, Malaysia

Pascaline Pré, Department of Energy Systems and Environment, GEPEA CNRS, IMT Atlantique, 44300, France

Lomig Hamon, Department of Environment and Energy Systems, GEPEA CNRS Unit, IMT Atlantique, 44307 Nantes, France

Azmi Mohd Shariff, Department of Chemical Engineering, Universiti Teknologi PETRONAS, 32610 Teronoh, Malaysia structures exist in form of linear, trigonal and imidazolate ligands. Strong connection between central metal ions and organic spacers creates diverse networks from which different type, shape and size of MOF can be produced.

However, the synthesis of these frameworks does not only involve the selection of connecting units for desired modules but also the affirmation of the ultimate configuration of the assembled building blocks [3]. Baerlocher et al. (2001) has introduced a structural entity called Secondary Building Unit (SBU) in the analysis of zeolites framework [4]. The SBU idea is then being adopted by other researchers in MOF studies to define the structure of the framework and determine its final coordination. SBU which is also known as paddlewheel is a 1Dchain of inorganic structure and their extended 3D-framework is formed with the help of organic bridging ligands. Containing magnesium as the central metal sites, a hexagonal Mg-MOF-74 is built from copolymerization of linear benzenedicarboxylate linker and 1D-helical channels of SBU $\left[\mathrm{Mg}_{3} \mathrm{O}_{3}\left(\mathrm{CO}_{2}\right)_{3}\right]_{\infty}$.

As suggested by different groups that worked for gas adsorption [5-8], graphene oxide could be introduced into MOF void spaces to enhance the formation of micropores in the framework for stronger retention of gaseous adsorbates. Of this study, new adsorbents were produced by doping GO into $\mathrm{Mg}$ MOF-74, designated as Mg-MOF-74@nGO composites where n is the percentage of substitution. Knowing that it is a challenge to understand the structure of non-typical materials, this paper intends to provide an indexing of Powder X-Ray Diffraction parameters of these composites. An investigation on $\left[\mathrm{Mg}_{3} \mathrm{O}_{3}\left(\mathrm{CO}_{2}\right)_{3}\right]_{\infty}$ structure has received less attention since engineering researchers have been focusing more on the adsorption performance of this material. The same investigation was earlier conducted by Ullah et al. (2013) for crystal structure of Pb-doped strontium ferrites [9].

\section{EXPERIMENTAL}

Solvothermal technique was used to synthesize $\mathrm{Mg}$ MOF-74 and GO composites. Magnesium nitrates, $\mathrm{Mg}\left(\mathrm{NO}_{3}\right)_{2} \cdot 6 \mathrm{H}_{2} \mathrm{O}$ and 2,5-dihydroxyterephtalic acid linker were completely dissolved in a solution of dimethylformamide/ethanol/deionized water by using ultrasonic agitation. Few drops of GO in water dispersion was added into the mixture before the agitation. This method was referred to in-situ synthesis of 'bottle-around-ship' method in which the composing material intercalates within the cages of MOF units [10]. The ultrasonication was carried out for hours to ensure complete dissolution of GO. 
The solution was then transferred into a Teflon lined stainless-steel autoclave reactor, tightly capped and left in the oven for $26 \mathrm{~h}$ at $125^{\circ} \mathrm{C}$ to let the reaction run under autogenous pressure. After that, the sample was removed and cooled at room temperature. The mother liquor of the reaction was gently separated from the product. The product was then immersed in methanol in a Petri dish to remove solvents blocked in the pores. The methanol was replenished 6 times for 3 days. After being separated, the wet powder was dried under mild temperature in the oven and the remaining solvent was evacuated under dynamic vacuum at $250^{\circ} \mathrm{C}$ for $15 \mathrm{~h}$. The composites were denoted as Mg-MOF-74@nGO ( $\mathrm{n}=0.1,0.2$, $0.3,0.4,0.5,0.6$ in wt \%). GO was measured in mass basis (wt\%) by referring to the mass of the pristine Mg-MOF-74 produced in prior synthesis. The crystalline structure of the samples was qualitatively confirmed by using PXRD. The patterns were recorded by using Bruker AXS D8 Advance Diffractometer with $\mathrm{Cu} \mathrm{K} \alpha(\lambda=1.5406 \AA)$ radiation, a step size of $0.02^{\circ}$ in $2 \theta$ ranged from $5^{\circ}$ to $50^{\circ}$, an exposure time of 100 $\mathrm{s} / \mathrm{step}$ and a scan rate of $0.1 \% \mathrm{~min}$.

\section{RESULTS AND DISCUSSION}

PXRD profile of the pristine Mg-MOF-74 $(n=0)$ is displayed in Fig 1 which is in perfect agreement with diffraction pattern calculated by Material Studio based on the structure from Cambridge Crystallographic Data Centre (database identifier FIJDOS, deposition number 265095), meaning that the pattern attributes to hexagonal crystallites $\left[\mathrm{Mg}_{3} \mathrm{O}_{3}\left(\mathrm{CO}_{2}\right)_{3}\right]_{\infty}$. The significant peaks are listed in Table 1.

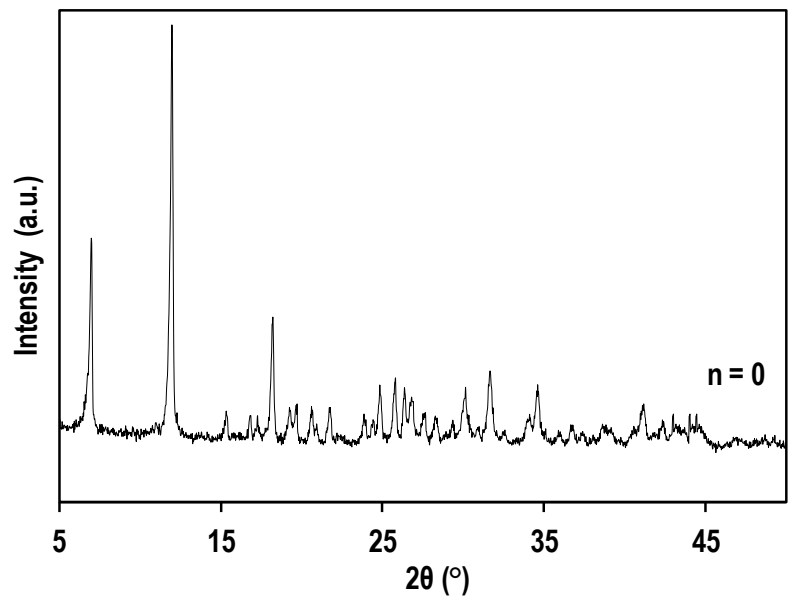

Fig 1. PXRD pattern of pristine Mg-MOF-74.

The first step involves indexing this pattern that will reveal the crystal system to which the material belongs. For that reason, the corresponding equations are presented as follows.

Hexagonal d-spacing formula [11]:

$$
\frac{1}{d^{2}}=\frac{4}{3} \frac{\left(h^{2}+h k+k^{2}\right)}{a^{2}}+\frac{l^{2}}{c^{2}}
$$

Bragg's law equation [11]:

$$
d=\frac{\lambda}{2 \sin \theta}
$$

Equation $1+$ Equation 2:

$$
\sin ^{2} \theta=A\left(h^{2}+h k+k^{2}\right)+C l^{2}
$$

$A$ and $C$ are constants:

$$
\begin{gathered}
A=\frac{\lambda^{2}}{3 a^{2}} \\
C=\frac{\lambda^{2}}{4 c^{2}}
\end{gathered}
$$

Where, $\lambda$ is incident $\mathrm{X}$-ray. $a$ and $c$ are lattice parameters of a hexagonal closed-pack unit that consists of four directions of a vertical $c$-axis (perpendicular to basal plane) and three horizontal $a_{1-3}$-axis (lying on basal plane).

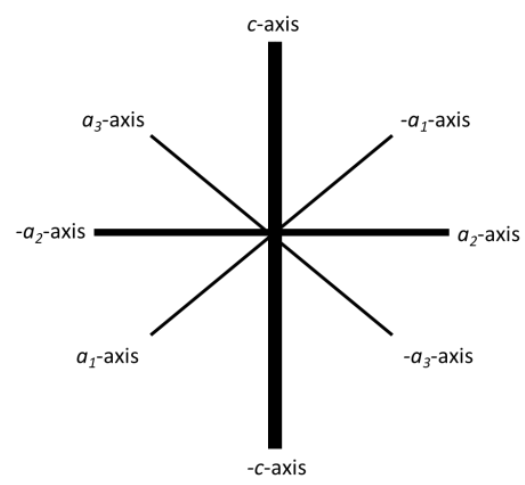

Where, $\lambda$ is incident $\mathrm{X}$-ray. a and $\mathrm{c}$ are lattice parameters of a hexagonal closed-pack unit that consists of four directions of a vertical c-axis (perpendicular to basal plane) and three horizontal a1-3-axis (lying on basal plane).

Equation 3 is developed by combining Bragg's law (Equation 2) and Equation 1 which is a modified form of cubic system's. This expression will subsequently determine Miller indices of non-cubic crystal system. The values of $A$ and $C$ in this relation are constant while $s=h^{2}+h k+k^{2}$ will be deduced by different $\sin ^{2} \theta$ values. As the pattern studied is expected to be hexagonal crystal structure, hence it should obey Equation 3 and the permissible values of $s$ are 1, 3, 4, 7, 9, etc. [12] Appendix 10. In this relation, the value of $A$ can be obtained at $h k O(l=0)$ while the value of $C$ can be obtained in the presence of $A$ and when $l \neq 0$. Therefore, as tabulated in Table 2, by assuming that $l=0$, the value of $\sin ^{2} \theta$ is divided by the $s$ values to calculate the constant $A$ from Equation 3 using the following expression:

$$
A=\frac{\sin ^{2} \theta}{h^{2}+h k+k^{2}}
$$

Then, the value of lattice parameter $a$ is calculated for all values of $\left(\sin ^{2} \theta\right) / s$ by using Equation 4 as shown in Table 3. In the table, there is some order in particular repetitive values which are being integral multiple of each other. For instance, 8.614538 is the multiplication of 4.322004 by integer 2 . The largest repetitive value is the lattice parameter of $a$ for 100 plane, $a / 2$ value for 200 plane, $a / 3$ value for 300 plane and etc. So that is how $h k 0$ indices of Peak 1 and Peak 5 are identified in Table 5. Knowing that $s=3$ and $l=0$, indices of Peak 4 can also be deduced.

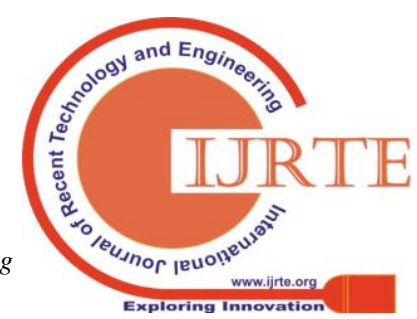


In contrary, the smallest repetitive value starred in Table 2 is considered to be $A$ which belongs to $a$ previously. Therefore, it can be concluded that the pristine Mg-MOF-74 exhibits lattice parameters values of $A=$ $0.010661 \AA$ and $a=8.614538 \AA$. The constant $C$ subsequently can be determined from Equation 3 by the following expression:

$$
C l^{2}=\sin ^{2} \theta-\left(h^{2}+h k+k^{2}\right) A
$$

For that reason, $A, 3 A, 4 A$ and $7 A$ are subtracted from $\sin ^{2} \theta$ which takes into account the permissible values of $s$. The results of $\mathrm{Cl}^{2}$ are tabulated in Table 4. Based on the table, there are four starred entries of interest $(0.007065$, $0.028295,0.063323$ and 0.112577 ) because they are in the ratio of integer $1,4,9$ and 16 . Therefore, it can be simplified as $0.007065=C(1)^{2}, 0.028295=C(2)^{2}, 0.063323=$ $C(3)^{2}$ and $0.112577=C(4)^{2}$. Immediately, $h k l$ indices of Peak 2, Peak 3, Peak 6 and Peak 7 can be identified knowing the corresponding value of $s$. The results are presented in Table 5. Thus, the pristine Mg-MOF-74 gives the values of $C=0.007065 \AA$ and $c=9.164558 \AA$. The same procedure was repeated for indexing pattern of all composites with different percentage of GO loading. The lattice parameters $a$ and $c$ for those samples are calculated. Consequently, the volume of unit cell and crystallite size are also calculated. The results are recapitulated in Table 6. The volume of unit cell, $V$, is calculated by following expression [13]:

$$
V=3^{1 / 2} \cdot a^{2} \cdot \frac{c}{2}=0.866 a^{2} \cdot c
$$

Crystallite size, D, for Peak 1 is calculated using Scherrer's formula [12]:

$$
D=\frac{k \cdot \lambda}{\beta \cdot \cos \theta}
$$

Where, $\mathrm{k}$ is a shape factor having a value of 0.94 for hexagonal crystal and $\beta$ is full width half maximum (FWHM) in radians.

Fig 3 represents the evolution of lattice parameters $a$ and $c$ with the increasing of GO composition in the materials. It can be noticed that the embedding of GO does not influence crystallites size in $a_{1-3}$-axis direction. However, there is an increasing progression in $c$-axis direction following a trend line of Equation 10. A hypothesis that can be deduced was the expansion of the lattices in $c$-axis direction due to strain contribution. The structural relaxation of the lattice was driven by the substitution of GO layers into the framework. The same distortion of lattices was also figured out by Yabuuchi et al. (2014) when doping over-sized gadolinium into gallium nitride films [14]. The calculated parameters of $c / a$ ratio, unit cell volume and Scherrer's crystallite size show that there is no significant influence of GO loading on those physical properties in the range of 0.1-0.6 wt $\%$.

$$
c=0.2114 n+9.2017, R^{2}=0.7334
$$

Table 1: Diffraction peaks and their corresponding angles taken from diffraction profile.

\begin{tabular}{ccc}
\hline Peak & $\mathbf{2 \theta}\left({ }^{\circ}\right)$ & $\boldsymbol{\theta}\left(^{\circ}\right)$ \\
\hline 1 & 11.8529 & 5.92645 \\
2 & 15.3019 & 7.65095 \\
3 & 19.3675 & 9.68375 \\
4 & 20.5484 & 10.27420 \\
5 & 23.7526 & 11.87630 \\
6 & 31.5667 & 15.78335 \\
7 & 41.1035 & 20.55175 \\
\hline
\end{tabular}

Table 2: Values of $\sin ^{2} \theta$ and their division by $s=1 ; 3 ; 4 ; 7$.

\begin{tabular}{ccccc}
\hline Peak & $\sin ^{2} \boldsymbol{\theta}$ & $\left(\sin ^{2} \boldsymbol{\theta}\right) / 3$ & $\left(\sin ^{2} \boldsymbol{\theta}\right) / 4$ & $\left(\sin ^{2} \boldsymbol{\theta}\right) / 7$ \\
\hline 1 & $0.010661^{*}$ & 0.003554 & 0.002665 & 0.001523 \\
2 & 0.017726 & 0.005909 & 0.004431 & 0.002532 \\
3 & 0.028295 & 0.009432 & 0.007074 & 0.004042 \\
4 & 0.031812 & $0.010604^{*}$ & 0.007953 & 0.004545 \\
5 & 0.042353 & 0.014118 & $0.010588^{*}$ & 0.006050 \\
6 & 0.073984 & 0.024661 & 0.018496 & $0.010569^{*}$ \\
7 & 0.123238 & 0.041079 & 0.030810 & 0.017605 \\
\hline
\end{tabular}

Table 3: Values of lattice parameter $a$ to find out $h k l$.

\begin{tabular}{ccccc}
\hline Peak & $\boldsymbol{a}_{(\boldsymbol{s}=\mathbf{1})}$ & $\boldsymbol{a}_{(\boldsymbol{s}=\mathbf{3})}$ & $\boldsymbol{a}_{(\boldsymbol{s}=\mathbf{4})}$ & $\boldsymbol{a}_{(\boldsymbol{s}=7)}$ \\
\hline 1 & $8.614538^{*}$ & 14.920817 & 17.229075 & $8.614538^{*}$ \\
2 & 6.680792 & 11.571472 & 13.361585 & 6.680792 \\
3 & 5.287836 & 9.158800 & 10.575671 & 5.287836 \\
4 & 4.986937 & $8.637628^{*}$ & 9.973873 & 4.986937 \\
5 & $4.322004^{*}$ & 7.485930 & $8.644007^{*}$ & $4.322004^{*}$ \\
6 & 3.270088 & 5.663958 & 6.540175 & 3.270088 \\
7 & 2.533706 & $4.388508^{*}$ & 5.067413 & 2.533706 \\
\hline
\end{tabular}

\begin{tabular}{|c|c|}
\hline Peak & h k l \\
\hline 1 & 100 \\
\hline 2 & 101 \\
\hline 3 & 002 \\
\hline 4 & 110 \\
\hline 5 & 200 \\
\hline 6 & 103 \\
\hline 7 & 004 \\
\hline
\end{tabular}

Table 4: Values of $\sin ^{2} \theta$ and subtraction of $s A$ with $s=1 ; 3 ; 4$.

\begin{tabular}{ccccc}
\hline Peak & $\sin ^{2} \theta$ & $\sin ^{2} \theta-A$ & $\sin ^{2} \boldsymbol{\theta}-3 \boldsymbol{A}$ & $\sin ^{2} \boldsymbol{\theta}-\mathbf{4 A}$ \\
\hline 1 & 0.010661 & & & \\
2 & 0.017726 & $0.007065^{*}$ & & \\
3 & $0.028295^{*}$ & 0.017634 & & \\
4 & 0.031812 & 0.021151 & & \\
5 & 0.042353 & 0.031692 & 0.010371 & \\
6 & 0.073984 & $0.063323^{*}$ & 0.042002 & 0.031341 \\
7 & 0.123238 & $0.112577^{*}$ & 0.091256 & 0.080595 \\
\hline
\end{tabular}

Table 5: Values of $h k l$ indices deduced from indexing.

Table 6: Characteristic parameters calculated for Mg-MOF$74 @$ nGO composites. $\mathrm{n}$ is graphene oxide composition in wt $\%$.

\begin{tabular}{clllll}
\hline $\mathbf{n}(\mathbf{w t} \boldsymbol{)})$ & $\boldsymbol{a}(\stackrel{\AA}{)})$ & $\boldsymbol{c}(\stackrel{\AA}{)})$ & $\boldsymbol{c} \boldsymbol{a}$ & $\boldsymbol{V}\left(\AA^{3}\right)$ & $\boldsymbol{D}(\mathbf{n m})$ \\
\hline 0 & 8.615 & 9.165 & 1.064 & 588.96 & 65.22 \\
0.1 & 8.741 & 9.247 & 1.058 & 611.91 & 60.53 \\
0.2 & 8.682 & 9.244 & 1.065 & 603.40 & 84.77 \\
0.3 & 8.732 & 9.310 & 1.066 & 614.70 & 84.76 \\
0.4 & 8.599 & 9.282 & 1.079 & 594.31 & 84.78 \\
0.5 & 8.633 & 9.285 & 1.076 & 599.22 & 60.54 \\
0.6 & 8.744 & 9.324 & 1.066 & 617.39 & 84.76 \\
\hline
\end{tabular}




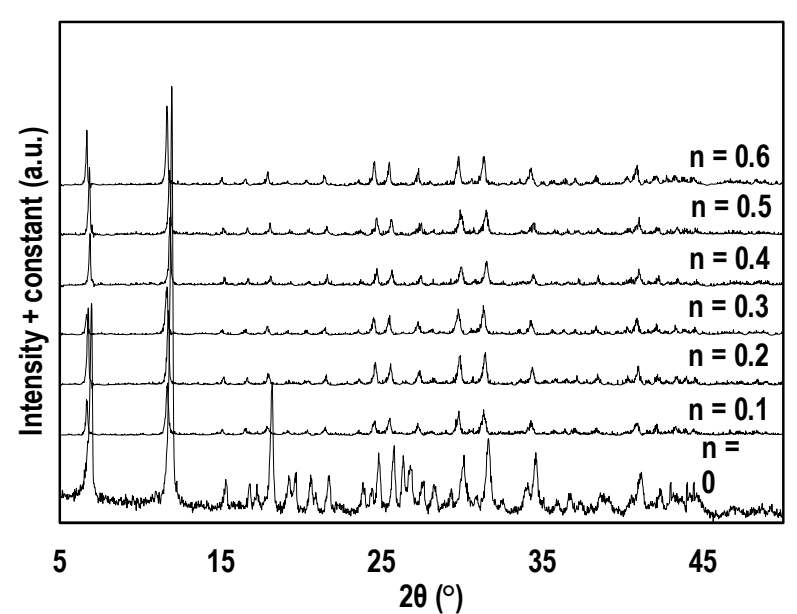

Fig 2. PXRD patterns of Mg-MOF-74@nGO composites. $n$ is graphene oxide composition in wt $\%$.

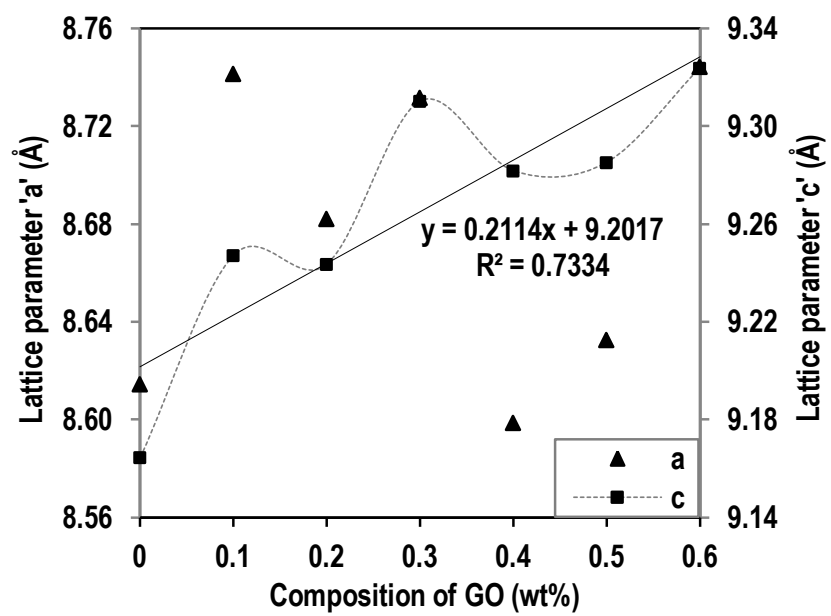

Fig 3. Lattice parameters of hexagonal crystallites of $\mathrm{Mg}$ MOF-74@nGO composites.

\section{REFERENCES}

[1] C. Dey, T. Kundu, B. P. Biswal, A. Mallick, and R. Banerjee, "CRYSTALLINE METAL-ORGANIC FRAMEWORKS (MOFS): SYNTHESIS, STRUCTURE AND FUNCTION," Acta Crystallographica Section B: Structural Science, Crystal Engineering and Materials, vol. 70, no. 1, pp. 3-10, 2014.

[2] P. Horcajada, C. Serre, M. Vallet-Regí, M. Sebban, F. Taulelle, and G. Férey, "METAL-ORGANIC FRAMEWORKS AS EFFICIENT MATERIALS FOR DRUG DELIVERY," Angewandte Chemie International Edition, vol. 118, no. 36, pp. 6120-6124, 2006.

[3] J. L. Rowsell and O. M. Yaghi, "METAL-ORGANIC FRAMEWORKS: A NEW CLASS OF POROUS MATERIALS," Microporous and Mesoporous Materials, vol. 73, no. 1, pp. 3-14, 2004.

[4] C. Baerlocher, D. H. Olson, and W. M. Meier, ATLAS OF ZEOLITE FRAMEWORK TYPES (FORMERLY: ATLAS OF ZEOLITE STRUCTURE TYPES). Elsevier, 2001.

[5] C. Petit, B. Levasseur, B. Mendoza, and T. J. Bandosz, "REACTIVE ADSORPTION OF ACIDIC GASES ON MOF/GRAPHITE OXIDE COMPOSITES," Microporous and Mesoporous Materials, vol. 154, pp. 107-112, 2012.

[6] C. Petit, B. Mendoza, and T. J. Bandosz, "HYDROGEN SULFIDE ADSORPTION ON MOFS AND MOF/GRAPHITE OXIDE COMPOSITES," ChemPhysChem, vol. 11, no. 17, pp. 3678-3684, 2010.

[7] Y. Chen, D. Lv, J. Wub, J. Xiao, H. Xi, Q. Xia, and Z. Li, “A NEW MOF-505@GO COMPOSITE WITH HIGH SELECTIVITY FOR $\mathrm{CO}_{2} / \mathrm{CH}_{4}$ AND $\mathrm{CO}_{2} / \mathrm{N}_{2}$ SEPARATION," Chemical Engineering Journal, no. 308, pp. 1065-1072, 2017.
[8] Y. Zhao, Y. Cao, and Q. Zhong, " $\mathrm{CO}_{2}$ CAPTURE ON METAL ORGANIC FRAMEWORK AND GRAPHENE OXIDE COMPOSITE USING A HIGH-PRESSURE STATIC ADSORPTION APPARATUS," Journal of Clean Energy Technologies, vol. 2, no. 1, pp. 34-37, 2014.

[9] Z. Ullah, S. Atiq, and S. J. Naseem, "INDEXING THE DIFFRACTION PATTERNS AND INVESTIGATING THE CRYSTAL STRUCTURE OF Pb-DOPED STRONTIUM FERRITES," Journal of Scientific Research vol. 5, no. 2, pp. 235$244,2013$.

[10] I. Ahmed and S. H. Jhung, "COMPOSITES OF METALORGANIC FRAMEWORKS: PREPARATION AND APPLICATION IN ADSORPTION," Materials Today, vol. 17, no. 3, pp. 136-146, 2014.

[11] V. Pecharsky and P. Zavalij, FUNDAMENTALS OF POWDER DIFFRACTION AND STRUCTURAL CHARACTERIZATION OF MATERIALS. Springer Science \& Business Media, 2008.

[12] B. D. Cullity, ELEMENTS OF X-RAY DIFFRACTION, 2nd ed. Department of Metallurgical Engineering and Materials Science, University of Nôtre Dame: Addison-Wesley Publishing Company Inc., 1978.

[13] S. Hussain and A. Maqsood, "INFLUENCE OF SINTERING TIME ON STRUCTURAL, MAGNETIC AND ELECTRICAL PROPERTIES OF Si-Ca ADDED Sr-HEXA FERRITES," Journal of Magnetism and Magnetic Materials, vol. 316, no. 1, pp. 73-80, 2007.

[14] A. Yabuuchi, N. Oshima, B. E. O'Rourke, R. Suzuki, K. Ito, S. Sano, K. Higashi, Y. K. Zhou, and S. Hasegawa, "STRUCTURAL AND DEFECT CHARACTERIZATION OF Gd-DOPED GaN FILMS BY X-RAY DIFFRACTION AND POSITRON ANNIHILATION," in Journal of Physics: Conference Series, vol. 505. IOP Publishing, p. 012023.

\section{AUTHOR PROFILE}

I am Khaliesah Kamal, currently I am affiliated with Department of Chemical Engineering, Universiti Teknologi PETRONAS, 31750 Seri Iskandar, Malaysia and Department of Energy Systems and Environment, IMT Atlantique, GEPEA CNRS, 44300 Nantes, France. My area of research is Metal-organic framework (MOF).

I am Mohamad Azmi Bustam, my current affiliation is Department of Chemical Engineering, Universiti Teknologi PETRONAS, 31750 Seri Iskandar, Malaysia. My area of research is Metal-organic framework (MOF).

I am Lomig Hamon, my current affiliation is Department of Environment and Energy Systems, GEPEA CNRS Unit, IMT Atlantique, 44307 Nantes, France. My area of research is Metal-organic framework.

I am Azmi Mohd Shariff, currently I am affiliated with Department of Chemical Engineering, Universiti Teknologi PETRONAS, 32610 Teronoh, Malaysia. My area of research is Metal-organic Framework 